\title{
Eosinophils and Neutrophils of Human Neonates Have Similar Impairments of Quantitative Up- regulation of Mac-1 (CD11b/CD18) Expression In Vitro ${ }^{1}$
}

\author{
JEFFREY B. SMITH, RAJU D. KUNJUMMEN, AND BHUPALA H. RAGHAVENDER \\ Department of Pediatrics, UCLA School of Medicine, UCLA Center for the Health Sciences, \\ Los Angeles, California 90024
}

\begin{abstract}
Neonatal neutrophils have Mac-1-related functional abnormalities that may contribute to the enhanced susceptibility of neonates to bacterial infections. We developed a flow cytometric method using differences in light scattering and CD16 expression to distinguish eosinophils from neutrophils and studied their responses to stimulation in vitro. Mean forward light scatter was $20-60 \%$ greater for adult neutrophils than eosinophils, $p$ $\leq 0.001$, and side scatter was $30-60 \%$ greater for eosinophils than neutrophils, $p \leq \mathbf{0 . 0 1}$, both before and after stimulation. Light scatter of neonatal and adult eosinophils did not differ. Mac-1 expression on adult eosinophils increased $60 \%$ after warming without specific stimulation and further increased 5-fold after incubation with $1 \mu \mathrm{M}$ A23187, $p<0.0001$. Platelet activating factor and recombinant C5a produced modest increases in eosinophil Mac1 expression, while $\mathbf{N}$-formyl-met-leu-phe and leukotriene B4 had minimal effects. Histamine and the eosinophil chemotactic factor of anaphylaxis tetrapeptides had no effect on either Mac-1 or CR1 (CD35) expression. After stimulation with $1 \mu \mathrm{M}$ A23187, eosinophils from eight healthy neonates at term expressed less Mac-1 than eosinophils from eight adults (mean $\pm \mathrm{SD}: 60.0 \pm 17.2$ versus $89.1 \pm 12.8, p=0.001$ ) by the same ratio as neonatal to adult neutrophils $(121.5 \pm 17.9$ versus $178.2 \pm 11.7, p=$ $0.0004)$. We conclude that neonatal eosinophils and neutrophils have similar impairments of quantitative Mac-1 up-regulation. Further studies are needed to determine if neonatal eosinophils also have functional abnormalities related to Mac-1. (Pediatr Res 30: 355-361, 1991)
\end{abstract}

\section{Abbreviations}

ECFA, eosinophil chemotactic factor of anaphylaxis FMLP, N-formyl-methionyl-leucyl-phenylalanine LTB4, leukotriene B4

PAF, platelet activating factor

PE, phycoerythrin

rC5a, recombinant C5a

Received February 13, 1991; accepted June 5, 1991

Correspondence and reprint requests: Dr. Jeffrey B. Smith, Department of Pediatrics, Rm. B2-325. UCLA Center for the Health Sciences, 10833 Le Conte Avenue, Los Angeles, CA 90024.

Supported in part by funds provided by the Committee on Research of the Academic Senate of the Los Angeles Division of the University of California and

by Cancer Center Core Grant CA-16042 from the National Institutes of Health.

'Presented in part at the Annual Meeting of the American Pediatric Society and the Society for Pediatric Research, May 1990.
Mac-1 is a leukocyte membrane glycoprotein with important functions in both phagocytosis and adherence. Although other cell-surface receptors also participate in these functions, the crucial role of Mac-1 and the other members of the CD11/CD 18 family in phagocyte function is demonstrated by patients with the leukocyte adhesion deficiency syndrome, in whom this family of molecules is congenitally absent. They suffer from recurrent bacterial infections, and their neutrophils have profound defects in adherence, chemotaxis, and C3bi-mediated phagocytosis (1, 2 ). The mobilization of blood leukocytes to a site of infection or inflammation in the tissues requires that the leukocytes first adhere to the endothelial lining of the blood vessel and then migrate through it. These processes involve interactions of leukocyte cell-surface receptors (including CDI la/CD18 and Mac1) with specific endothelial ligands and are regulated by the effects of inflammatory mediators on both the leukocyte and the endothelial cell $(3,4)$. The quantitative expression of CDI1a/ CD18 (LFA-1) on neutrophils does not change in response to stimulation, but the expression of Mac-1 (and of CD11c/CD18) increases dramatically because of rapid translocation from an intracellular storage pool to the plasma membrane surface $(2,5$, 6). In addition, stimulation of neutrophils produces qualitative changes in Mac-1-mediated functions (3, 7). It has been suggested that quantitative up-regulation may be more important in chemotaxis and phagocytosis than in adherence (2).

Bacterial infection continues to be a major problem of newborn infants, especially those delivered prematurely. Deficiencies in neutrophil function are among the developmental abnormalities thought to contribute to the infectious susceptibility of the neonate, inasmuch as neonatal neutrophils have marked impairments in adherence and chemotaxis, as well as subtler defects in phagocytosis and bactericidal capacity $(8,9)$. Abnormalities in Mac-1-related functions of neonatal neutrophils may have an important role in their adherence-related defects. Neutrophils from healthy human neonates at term express less Mac- 1 in response to stimulation than do neutrophils from adults $(10,11)$, and neutrophils from preterm infants have a further diminution of Mac-1 up-regulation compared with neutrophils of term infants (12). In isolation, these differences in quantitative expression are unlikely to be significant, since the maximum levels of Mac-1 on neonatal neutrophils are comparable to those on neutrophils of leukocyte deficiency syndrome heterozygotes, who have no increased risk of infection. However, Anderson et al. $(11,13)$ have demonstrated that neonatal neutrophils do have Mac-1-related impairments in stimulated adherence and transendothelial migration. The effects on neonatal neutrophils of the quantitative and qualitative Mac-1-related abnormalities may be magnified by abnormalities of other adherence receptors (14).

The study of eosinophils may provide insight into mechanisms that they share with neutrophils and also illuminate the differences between these closely related types of cells. Mac-1 is 
expressed on eosinophils and is up-regulated in response to stimulation (15-19), and stimulation of eosinophils produces increased adherence, which is Mac-1-dependent in part $(20,21)$. There have been no studies of Mac-1 expression on neonatal eosinophils, and it is not known whether neonatal eosinophils have functional abnormalities. To determine whether neonatal eosinophils have a quantitative impairment in Mac-1 up-regulation similar to that of neonatal neutrophils, we developed a method for measuring Mac-1 on neutrophils and eosinophils in mixed leukocyte suspensions using dual-labeling with an antiCD16 MAb together with light-scatter gating and studied the responses of adult and neonatal eosinophils to stimulation in vitro.

\section{MATERIALS AND METHODS}

Subjects. Blood was obtained from the placental side of the umbilical cord immediately after uncomplicated vaginal or cesarean deliveries of healthy infants at term gestation. Blood from healthy adult volunteers was drawn from a peripheral vein. Specimens were obtained, with appropriate consent, in accordance with an approved institutional protocol.

Materials. Except as specified, the buffer used in all experiments was Hanks' balanced salt solution, without $\mathrm{Ca}, \mathrm{Mg}$, bicarbonate, or phenol red, supplemented with $2 \%$ by volume heatinactivated FCS (GIBCO, Grand Island, NY). Stock solutions were adjusted to $\mathrm{pH} 7.35-7.40$, passed through a $0.22-\mu \mathrm{m}$ filter, and maintained sterile at $4^{\circ} \mathrm{C}$.

Calcium ionophore A23187, FMLP, human rC5a, PAF, LTB4, histamine, and ECFA tetrapeptides ala-gly-ser-glu and val-gly-ser-glu were obtained from Sigma Chemical Co. (St. Louis, MO).

MAb conjugated with PE or FITC were obtained from Becton Dickinson (Mountain View, CA). PE-conjugated anti-Leu-15 (anti-CD11b), FITC-conjugated anti-Leu-1 la (anti-CD16), and the corresponding PE- and FITC-conjugated isotype-specific controls were used. Anti-CR1 MAb (anti-CD35) and an unlabeled $\mathrm{IgG}_{1}$ control (Becton Dickinson) were used in a few experiments and were indirectly labeled using PE-conjugated goat anti-mouse $\mathrm{F}\left(\mathrm{ab}^{\prime}\right)_{2}$ antibodies (Tago, Inc., Burlingame, CA). Fluorescent beads from Coulter Corp. (Hialeah, FL) $(10 \mu \mathrm{m}$ diameter, $2 \%$ bright) and from Flow Cytometry Standards Cor poration (Research Triangle Park, NC) were used in each experiment as fluorescence and light-scattering references.

Cell processing. Blood samples were transferred to EDTAanticoagulated tubes and placed on ice immediately after collection. An adult specimen was obtained at the same time as each neonatal specimen and processed in parallel. Except as specified, samples were maintained at $0-4^{\circ} \mathrm{C}$ throughout the procedure, which was similar to one that we described previously (12). Briefly, erythrocytes were removed by hypotonic lysis, and aliquots of the leukocyte suspension containing $10^{5}$ polymorphonuclear cells were incubated for $20 \mathrm{~min}$ at $37^{\circ} \mathrm{C}$ with specified concentrations of a stimulating factor in buffer containing $\mathrm{Ca}$ and $\mathrm{Mg}$. Parallel tubes were kept on ice $\left(0^{\circ} \mathrm{C}\right.$ control) or incubated with buffer alone $\left(37^{\circ} \mathrm{C}\right.$ control). After the incubation, the tubes were placed back on ice and cold buffer containing $0.1 \%$ sodium azide was added to each tube. The cells were washed, resuspended in $0.1 \mathrm{~mL}$ of the azide buffer, and double-labeled by incubation for $30 \mathrm{~min}$ in the dark at $0^{\circ} \mathrm{C}$ with saturating amounts of FITCconjugated Leu-11a and PE-conjugated anti-Leu-15 or with control antibodies. After a final wash to remove unbound antibody, the cells were resuspended in $0.3 \mathrm{~mL}$ of azide buffer for flow cytometric analysis. Differential counts of Wright-stained smears of whole blood and of cytocentrifuge preparations of the leukocyte suspensions were made for each specimen.

Alternative preparations. In preliminary experiments, the results for cells prepared by the method described above were compared with two methods that did not utilize hypotonic lysis of erythrocytes. First, stimulation and labeling were performed on aliquots of washed whole blood, followed by incubation for 10 min at room temperature with FACS lysing solution (Becton Dickinson). The leukocytes were then washed and resuspended in cold buffer for analysis. Second, whole blood was centrifuged at $400 \times g$ for $25 \mathrm{~min}$ at $4^{\circ} \mathrm{C}$ on discontinuous gradients of 65 and $72.5 \%$ isotonic Percoll (Pharmacia, Piscataway, NJ). Leukocyte suspensions were obtained sufficiently free of erythrocytes to allow stimulation, labeling, and analysis without any lysis procedure. In other studies, we compared our standard procedure with granulocyte suspensions of neonatal blood subjected to a preliminary centrifugation through $65 \%$ Percoll at $4^{\circ} \mathrm{C}$ to remove mononuclear cells and light-density neutrophils.

Eosinophil purification. For comparison with the results of the gating procedure, purified eosinophils were obtained using a modification of the procedure of Roberts and Gallin (22). After incubation at $37^{\circ} \mathrm{C}$ for $30 \mathrm{~min}$ with $1 \mu \mathrm{M}$ FMLP, EDTAanticonjugated whole blood was centrifuged at $400 \times g$ for 25 min on a discontinuous density gradient of 62.5 and $72.5 \%$ Percoll at room temperature, and the eosinophil layer was collected and washed. An aliquot of blood was kept at $0^{\circ} \mathrm{C}$ as a control. After hypotonic lysis of erythrocytes, aliquots of the purified eosinophils and the control leukocytes were incubated with stimulating factors and double-labeled with the MAb as described above.

Cell analysis. Flow cytometry was performed on a FACScan (Becton Dickinson), with the same photomultiplier voltages used in all experiments. Color compensation settings were verified by comparison of the red and green fluorescence values of unlabeled, single-labeled, and double-labeled neutrophils. Logarithmic amplification was used for the green fluorescence channel (CD16), which was used only for gating. Red fluorescence data (Mac-1) were collected with linear amplification, and the gain needed to place the relevant distribution on scale was recorded. The mean linear fluorescence (MLF) of aliquots from a single lot of the Coulter Corp. beads was measured in each experiment and used as a reference standard. Mac-1 expression, defined as the specific mean relative fluorescence of a cell population, was calculated as a percentage of the reference bead fluorescence according to the formula

$100 \times($ sample MLF/sample gain $)$

$$
\times(\text { bead } M L F / \text { bead gain })^{-1}-\mathrm{RF}_{0}
$$

where $\mathrm{RF}_{0}$ was the relative fluorescence of the cells labeled with the control MAb. The control antibody fluorescence values were consistently very low $(0.0-0.2$ for neutrophils and $0.1-0.4$ for eosinophils) compared with the values for the specific antibodylabeled cells after stimulation as well as in the 0 and $37^{\circ} \mathrm{C}$ controls. Light-scatter data were also collected with linear amplification and were normalized in a similar manner to the values for the reference beads in each experiment.

Two list-mode data sets were acquired for each specimen. First, 5000 events were collected with the red-fluorescence gain optimal for the neutrophil Mac-1 distribution, with gates for the forward versus side-scatter distribution drawn to exclude mononuclear cells. Basophils also fell in this excluded region (23). Data for neutrophils were subsequently analyzed by gating the CD16-bright cells together with a refined light-scatter gate. Because eosinophils were a small minority of the granulocytes in most specimens, a second data set of approximately 1000 events was collected with a gate excluding the CD16-bright cells (neutrophils) and with the red-fluorescence gain optimized for eosinophils. As shown below, the light-scatter distributions of eosinophils and neutrophils were distinguishable. Data for eosinophils were obtained by gating for the CD16-dim peak together with a gate encircling the eosinophil region in the light scatter distribution of the CD16-dim cells. Because the light-scatter distributions of both neutrophils and eosinophils changed after stimulation, the positions of the scatter gates were checked for each specimen and adjusted as needed. For the illustrations, smoothed contour 
plots (at $15 \%$ linear intervals) were made with the Lysis program (Becton Dickinson).

Statistics. The mean values of fluorescence and light scatter distributions of individual cell populations were calculated with the FACScan Research Software, version B (Becton Dickinson). Group statistics were computed with the StatView $512^{+}$program (Brainpower Inc., Calabasas, CA) for the Macintosh computer (Apple Computer, Cupertino, CA) and results were expressed as the group means $\pm \mathrm{SD}$ of the individual distribution mean values. Repeated-measures analysis of variance was used to test for differences among the subject groups (adult and neonatal) and cell types (neutrophil and eosinophil). When differences were found by analysis of variance $(p \leq 0.05)$, cell values for individual conditions were compared by the $t$ test, with $p \leq 0.01$ as the criterion for significance.

\section{RESULTS}

CD16 and light-scatter distributions. Histograms of CD16 expression (Leu-11a fluorescence) and contour plots of the lightscatter distributions of four illustrative specimens are shown in Figure 1. CD16 expression of purified eosinophils was minimal compared with that of neutrophils, and the eosinophil and neutrophil CD16 peaks remained well separated after stimulation with FMLP or A23187. The light-scattering properties of eosinophils and neutrophils were recognizably different in all specimens, with the eosinophil distribution centered at larger sidescatter values and smaller forward-scatter values than neutrophils. The separation of the eosinophil and neutrophil lightscatter peaks increased after stimulation.

The light-scattering distributions of eight pairs of adult and neonatal specimens were analyzed in detail (Fig. 2). In the adult specimens, the mean forward scatter of neutrophils exceeded that of eosinophils by about $20 \%$ in the 0 and $37^{\circ} \mathrm{C}$ controls, $p<$ 0.001 , and after stimulation with FMLP or A23187 increased to levels $40-60 \%$ greater than those of the eosinophils in the same specimens, $p<0.0001$. The mean side scatter of eosinophils was $30-60 \%$ greater than that of neutrophils in all conditions, $p \leq$ 0.01 . In the neonatal specimens, the relationship of neutrophil to eosinophil light scatter was similar to the adult relationship, with differences significant at $p<0.005$ except for forward scatter at the two highest concentrations of A23187. Forward light scatter of the neonatal neutrophils was $10 \%$ greater than for adult neutrophils in the $37^{\circ} \mathrm{C}$ controls, but was $10-20 \%$ less than the adult neutrophil forward light scatter at the two highest A23187 concentrations, $p \leq 0.001$. Side scatter of neonatal and adult neutrophils did not differ, and there were no differences in light scattering of the neonatal and adult eosinophils.

Comparison with purified eosinophils. The Mac-1 fluorescence
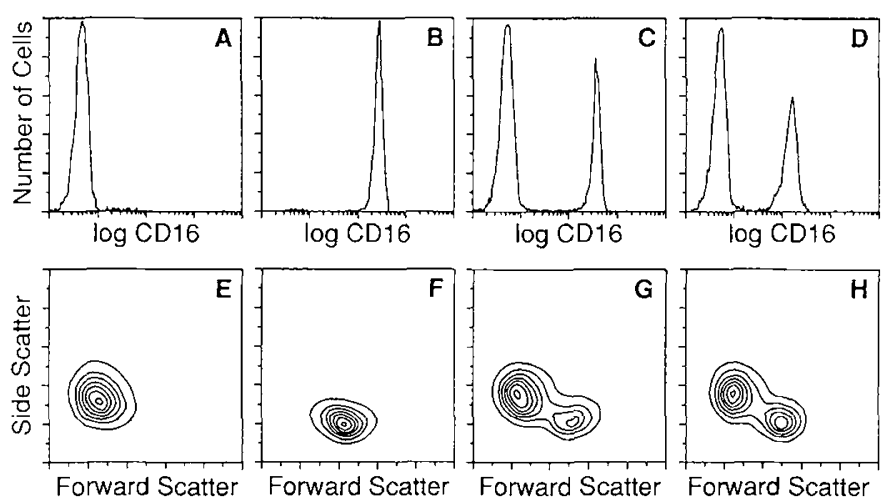

Fig. 1. CD16 fluorescence distributions and contour plots of the lightscatter distributions of four illustrative specimens. $A, E,>99 \%$ purified eosinophils; $B, F$, unstimulated granulocytes from whole blood, containing $>98 \%$ neutrophils; $C, G$, a mixture containing $69 \%$ eosinophils and $30 \%$ neutrophils, after stimulation with $1 \mu \mathrm{M}$ FMLP; $D, H$, the same mixture after stimulation with $1 \mu \mathrm{M}$ A23 187.
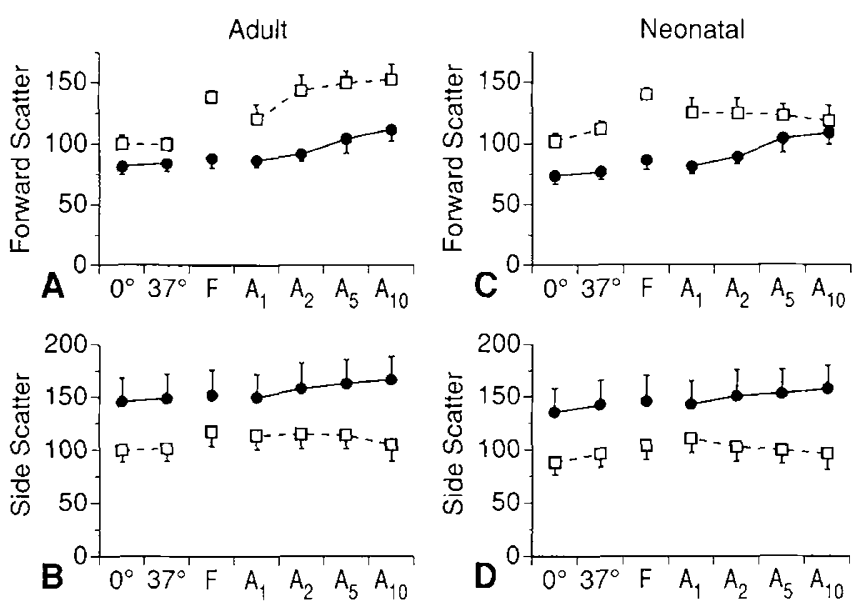

Fig. 2. Relative light scatter of adult and neonatal granulocytes. Forward and side scatter of neutrophils (open squares) and eosinophils (solid circles) were determined by CD 16 gating of control specimens ( 0 and $37^{\circ} \mathrm{C}$ ) and of specimens incubated at $37^{\circ} \mathrm{C}$ for $20 \mathrm{~min}$ and $1 \mu \mathrm{M}$ FMLP $(F)$, or with $\mathrm{A} 23187$ at $125,250,500$, or $1000 \mathrm{nM}\left(A_{1}, A_{2}, A_{5}\right.$, and $A_{10}$, respectively). Data are the group means $\pm \mathrm{SD}$ of the individual distribution means for eight pairs of adult $(A, B)$ and neonatal $(C, D)$ specimens.
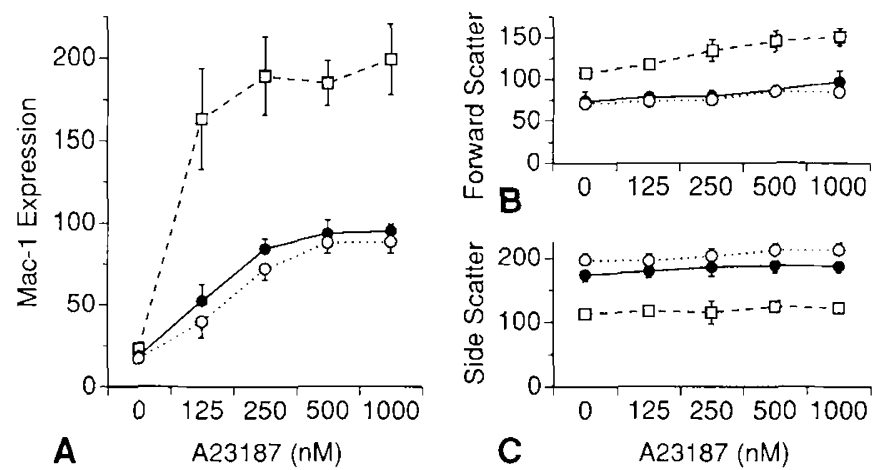

Fig. 3. Comparison of Mac- 1 expression and light scatter of purified eosinophils (open circles) with the results of the gated analysis for eosinophils (solid circles) and neutrophils (open squares). Data are the means \pm SD for three adult specimens.

and light scatter properties of eosinophils in mixed leukocyte suspensions analyzed by the double-gating procedure were compared with those of eosinophils purified by the method of Roberts and Gallin (22), which uses preincubation with FMLP to induce a density change in neutrophils, followed by Percoll density gradient centrifugation. A23187-stimulated expression of Mac-1 on eosinophils in the normal mixed-leukocyte specimens was in close agreement with that of the purified eosinophils (Fig. 3A) and was less than half that of A23187-stimulated neutrophils. Neutrophils preincubated with FMLP and then stimulated with A23187 (not shown) had 10-20\% greater Mac-1 expression than neutrophils not preincubated with FMLP. The mean light-scatter values of the purified eosinophils were close to the eosinophil values obtained by the gated analysis and were distinctly different from the light-scatter values of neutrophils (Fig. $3 B$ and $C$ ). Similar agreement between purified eosinophils and the results of the gated analysis was obtained for neonatal blood specimens (not shown).

Other control studies. Preliminary studies were performed to assess the effects of different methods of erythrocyte removal. We found no differences in Mac-1 expression, before or after stimulation, between neutrophils or eosinophils briefly subjected to hypotonic saline for erythrocyte lysis, compared with cells from the same donors separated from erythrocytes by a preliminary centrifugation on Percoll. Mac-1 expression also did not 
differ in comparison with whole blood specimens stimulated and labeled with antibodies before erythrocyte removal using FACS lysing solution. However, this method (which also fixes the leukocytes) resulted in a much greater overlap of the light-scatter distributions of neutrophils and eosinophils than was the case for unfixed cells.

Incubation of leukocyte suspensions with FMLP, A23187, rC5a, PAF, or LTB4 resulted in prompt increases in neutrophil Mac- 1 expression, which reached maximal levels by $20 \mathrm{~min}$. The time course of eosinophil Mac-1 up-regulation was similar, and there were no significant changes when the incubations were continued for up to $60 \mathrm{~min}$ (not shown).

In a few cord blood specimens, the light-scatter distributions showed a small subpopulation of CD16-dim cells with light scattering consistent with neutrophils and distinct from the eosinophil peak. These cells were not included in our analysis of either eosinophils (excluded by the light-scatter gate) or neutrophils (excluded by gating the CD16-bright peak). For the eight pairs of specimens used for the light-scattering data in Figure 2, this excluded group of cells numbered only $0.2 \pm 0.1 \%$ of adult neutrophils and $2.4 \pm 1.6 \%$ of neonatal neutrophils. This subpopulation was eliminated by a preliminary centrifugation of the neonatal blood through $65 \%$ Percoll at $4^{\circ} \mathrm{C}$, indicating that it was composed of light-density cells. Results for Mac-1 expression of the CD16-dim cells in these preparations were identical to the double-gated analysis of eosinophils in the whole-blood preparation from the same specimens (not shown).

Response to warming. The expression of $\mathrm{Mac}-1$ and other membrane proteins on neutrophils may increase significantly during in vitro procedures unless the cells are kept cold $(6,24)$. To determine whether a similar phenomenon occurs for eosinophils, cells kept on ice were included in each experiment for comparison with the cells incubated in buffer at $37^{\circ} \mathrm{C}$ (Fig. 4). Eosinophil expression of Mac-l increased significantly after warming in both adult and neonatal specimens, $p \leq 0.0001$. However, the magnitude of the increase, about $60 \%$, was considerably less than the increase in neutrophils. Because of this, the relationship of eosinophil to neutrophil $\mathrm{Mac}-1$ expression changed after the cells were warmed. In the $0^{\circ} \mathrm{C}$ controls, eosinophil Mac-1 was nearly double that of the neutrophils in the adult specimens ( $11.4 \pm 2.4$ versus $6.4 \pm 3.4, p<0.0001$ ), but was only slightly larger than neutrophil Mac-1 in the neonatal specimens $(11.1 \pm 2.7$ versus $9.1 \pm 2.7, p=0.02)$. After incubation at $37^{\circ} \mathrm{C}, \mathrm{Mac}-1$ expression of neonatal neutrophils exceeded that of neonatal eosinophils $(25.9 \pm 7.8$ versus $17.7 \pm 3.2, p=$ 0.0002 ), while Mac-1 expression of adult neutrophils did not differ from that of eosinophils $(19.8 \pm 6.3$ versus $18.1 \pm 4.1, p$ $=0.16$ ). Mac- 1 expression of neonatal and adult eosinophils did not differ in either the $0^{\circ} \mathrm{C}$ controls or after incubation at $37^{\circ} \mathrm{C}$. In contrast, Mac-1 expression of the neonatal neutrophils was $42 \%$ greater than adult neutrophil values before and $31 \%$ greater after incubation at $37^{\circ} \mathrm{C}$ ( $p=0.05$ and 0.01 , respectively).

A23187 stimulation. The effect of incubation with A23187 on eosinophil and neutrophil Mac-1 expression was determined for eight pairs of adult and neonatal specimens (Fig. 5). Eosinophil
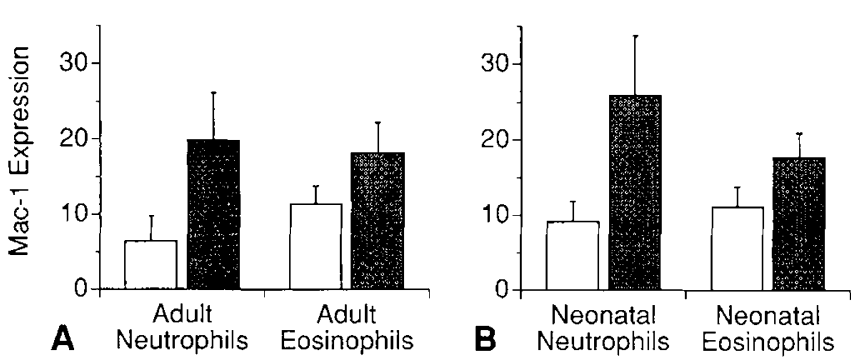

Fig. 4. Effect of warming on Mac-1 expression. Neutrophils and eosinophils were maintained at $0^{\circ} \mathrm{C}$ (open bars) or incubated in buffer at $37^{\circ} \mathrm{C}$ for $20 \mathrm{~min}$ (shaded bars) before labeling with antibody. Data are the means $\pm S D$ for 18 pairs of adult and neonatal specimens.
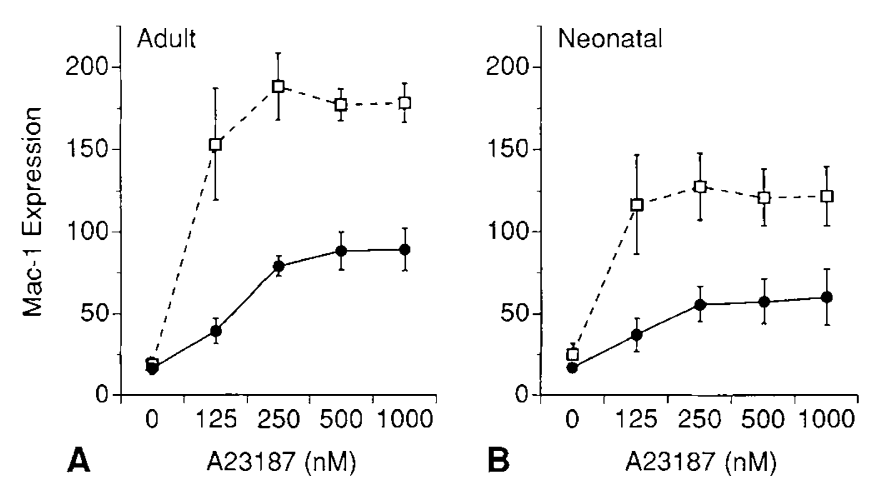

Fig. 5. Up-regulation of Mac-1 on eosinophils (solid cirlces) and neutrophils (open squares) after incubation at $37^{\circ} \mathrm{C}$ for $20 \mathrm{~min}$ with increasing concentrations of $\mathrm{A} 23187$. Data are the means $\pm \mathrm{SD}$ for eight pairs of adult and neonatal specimens.
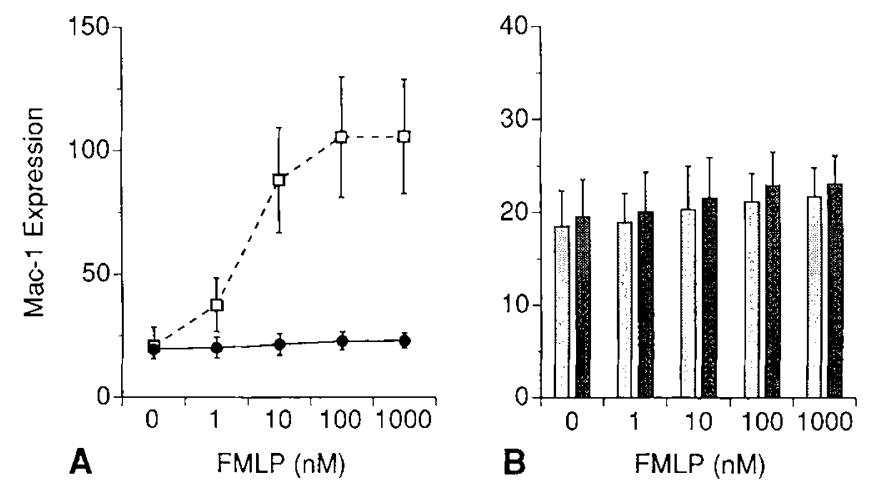

Fig. 6. Mac-1 expression (mean \pm SD) after incubation with FMLP at $37^{\circ} \mathrm{C}$ for $20 \mathrm{~min}$. $A$, eosinophils (solid circles) and neutrophils (open squares) from 10 adults. $B$, eosinophils from 10 neonates (light bars) and 10 adults (dark bars).

Mac-1 expression increased substantially after incubation with A23187 at $125 \mathrm{nM}$ or more, $p \leq 0.0004$. The maximal values were 3.5 -fold greater for neonatal eosinophils and 5.4-fold greater for adult eosinophils than controls incubated in buffer alone. For neutrophils, maximal Mac-1 expression was 4.8-fold greater for neonatal cells and 9.5-fold greater for adult cells, relative to the respective controls. For both neonatal and adult specimens, Mac1 expression on eosinophils was significantly less than that on neutrophils at each concentration of A23187 tested, $p \leq 0.0001$ Maximal Mac-1 expression of adult eosinophils, at $1 \mu \mathrm{M}$ A23187, was $50 \%$ of the values for adult neutrophils $(89.1 \pm 12.8$ versus $178.2 \pm 11.7$ ), and maximal Mac-1 expression of neonatal eosinophils was $49 \%$ of the neonatal neutrophil values $(60.0 \pm 17.2$ versus $121.5 \pm 17.9$ ). Relative to the values for the adult cells, therefore, maximal A23187-stimulated Mac-1 expression on neonatal eosinophils was nearly identical to that of neonatal neutrophils.

After incubation with $\mathrm{A} 23187$ at $250 \mathrm{nM}$ or more, neonatal eosinophils expressed less Mac- 1 than eosinophils from adults, $p$ $\leq 0.001$, with values averaging $67 \%$ of those of adult eosinophils. This was strikingly similar to the results for neonatal neutrophils, which also expressed less Mac- 1 than adult neutrophils after incubation with $\mathrm{A} 23187$ at these concentrations, $p<0.001$, with values averaging $68 \%$ of the adult neutrophil values.

FMLP stimulation. The response of eosinophils to incubation with FMLP differed markedly from that of neutrophils (Fig. $6 \mathrm{~A}$ ). Adult neutrophil Mac-1 expression increased after incubation with $1 \mathrm{nM}$ or more FMLP, $p \leq 0.0002$, to maximal values 5.1fold greater than the control neutrophils. Adult eosinophil Mac1 expression also increased with incubation with FMLP at concentrations of $10 \mathrm{nM}$ or greater, $p<0.01$, but the increase was trivial compared with that of neutrophils. As shown in Figure 
$6 B$, there was no difference in the response of adult and neonatal eosinophils to incubation with FMLP ( $p=0.47$ by repeatedmeasure ANOVA). Mac-1 expression of neonatal neutrophils (not shown) was significantly less than that of the adult neutrophils after incubation with FMLP at 10 nM FMLP or more, $p \leq$ 0.01 . Maximal values, at $1 \mu \mathrm{M}$ FMLP, averaged $70 \%$ of the adult neutrophil mean, in close agreement with published values for term infant neutrophils (10-12).

Other stimulating factors. The responses of eosinophils and neutrophils to a number of other potential activators were studied. Figure 7 shows the differing responses of neutrophils and eosinophils to increasing doses of rC5a, PAF, and LTB4. Each of these substances produced substantial up-regulation of Mac-1 on neutrophils. In contrast, Mac-1 expression on eosinophils increased only modestly after incubation with PAF and $\mathrm{rC5a}$, and LTB4 had a minimal effect. The maximal effect of zymosanactivated serum (not shown) was similar to that of rC5a. Histamine (at $1-100 \mu \mathrm{M}$ ) and the ECFA tetrapeptides (at $10 \mathrm{nM}-100$ $\mu \mathrm{M})$ had no effect on neutrophil or eosinophil expression of either Mac-1 or of CR 1 after incubation at $37^{\circ} \mathrm{C}$ for either 30 or $60 \mathrm{~min}$. In the same experiments, CR1 and Mac-1 expression increased 3.4- and 3.9-fold, respectively, on eosinophils and 5.0and 9.0-fold on neutrophils after incubation with $500 \mathrm{nM}$ A23187.

\section{DISCUSSION}

We studied the up-regulation of Mac-1 expression on neonatal and adult eosinophils, using a method that allowed comparison with neutrophils in the same specimens under identical conditions. We found that A23187-stimulated up-regulation of Mac1 on neonatal eosinophils was diminished relative to adult eosinophils to precisely the same extent as for neutrophils. This suggests that the underlying mechanisms, which are unknown, may be the same for both cell types. In neutrophils, the abnormality involves an impairment of mobilization of the Mac-1 from the intracellular pool, since the total cellular content of Mac- 1 in adult and neonatal neutrophils does not differ (25). Neither the location nor size of the intracellular pool of Mac-1 has been studied in either adult or neonatal eosinophils. Indeed, information about most properties of neonatal eosinophils is scanty, and it is not known if neonatal eosinophils have functional defects similar to those of neonatal neutrophils. Further investigation of neonatal eosinophils as well as neutrophils may help to illuminate the mechanisms that underlie neonatal granulocyte abnormalities.

Our interest in eosinophils was stimulated by the observation that eosinophils constituted an unexpectedly large proportion of the granulocytes in fetuses with $\mathrm{Rh}$ disease at 20-30 wk gestation (12). Eosinophil counts in normal infants are higher than in adults, and preterm infants frequently develop a significant eosinophilia $(26,27)$, but the function of eosinophils in neonatal host defense and inflammation is not known. Regardless of their function, the frequent occurrence of significant numbers of

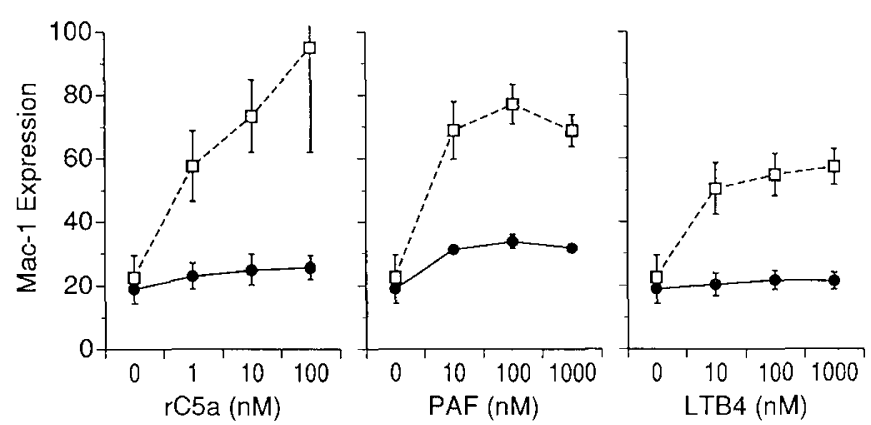

Fig. 7. Mac-1 expression of eosinophils (solid circles) and neutrophils (open squares) after incubation with rC5a, PAF, or LTB4 for 20 min at $37^{\circ} \mathrm{C}$. Data are mean $\pm \mathrm{SD}$ for two adult specimens. eosinophils in fetal and neonatal blood implies that studies of neonatal granulocytes will often require methods that can distinguish or separate eosinophils from neutrophils.

Compared with eosinophils, neutrophils express large amounts of CD16, the type III $\mathrm{Fc}_{\gamma}$ receptor (28-30), and this provides a way to distinguish or isolate eosinophils from neutrophils by negative selection. Highly purified preparations of eosinophils were obtained by Hansel et al. (31), using anti-CD16 MAbcoated magnetic beads to remove neutrophils, and Hartnell et al. (17) used an anti-CD16 MAb to exclude residual neutrophils in flow cytometric studies of purified eosinophils. We found that the light-scatter distributions of eosinophils and neutrophils were sufficiently well separated to make light-scatter gating a useful adjunct to CD16 expression in distinguishing these cells in mixed leukocyte suspensions, both before and after stimulation (32). A similar observation (for unstimulated specimens) was recently reported by Terstappen et al. (23). We verified our gating procedure by direct comparison with the results for Mac-1 expression and light scattering of purified eosinophils.

The use of CD16 MAb to distinguish eosinophils from neutrophils may be advantageous when limited quantities of blood are available, as is often the case for neonates, and when it is important to avoid the effects of isolation procedures that can alter cell properties or select nonrepresentative subpopulations (33). The method is subject to some limitations, however. First, it should be noted that some anti-CD16 antibodies are specific for one form of this polymorphic receptor and would not be suitable (34). The antibody we used (anti-Leu-11a) reacts with neutrophils from all individuals. Second, CD16 can be shed from the neutrophil surface in vivo or in vitro after activation (35-37). To minimize in vitro shedding of CD16, we found it important to begin our experiments as soon as the blood specimens were collected and to complete the flow cytometric analysis within about $8 \mathrm{~h}$.

The increase in Mac-1 expression that we found when eosinophils were incubated at $37^{\circ} \mathrm{C}$ in the absence of any specific stimulation is similar to the behavior previously observed for neutrophil CR1 and Mac-1 $(6,24)$. This indicates that receptor expression on eosinophils as well as neutrophils is susceptible to nonspecific up-regulation during in vitro procedures. Previous studies of eosinophil Mac-1 expression have used methods involving incubation at $37^{\circ} \mathrm{C}$ as part of the purification procedure (15-19). This probably accounts for the finding of Thorne et al. (19) that CD 11 b expression was higher on neutrophils than eosinophils before specific stimulation. We found, in contrast, that Mac-1 expression of eosinophils was nearly twice that of neutrophils in adult blood specimens kept at $0-4^{\circ} \mathrm{C}$.

In both 0 and $37^{\circ} \mathrm{C}$ control specimens, Mac-1 expression of neonatal neutrophils was greater than that of adult neutrophils in the corresponding condition. This is consistent with reports indicating an activating or priming effect on oxidative and other responses of cord blood neutrophils $(10,38,39)$. The increased forward light scatter of neonatal neutrophils at $37^{\circ} \mathrm{C}$ and the progressive decrease in their forward scatter with increasing concentrations of A23187 are also consistent with a priming effect (40). In contrast, there were no differences between neonatal and adult eosinophils in Mac-1 expression or light scatter. This suggests that eosinophils may be insensitive to the factor or factors that cause priming of cord blood neutrophils during labor and delivery.

In this study, as in previous studies (11), neutrophil Mac-1 expression was markedly increased by a variety of stimulating factors, including the chemotactic factors C5a and FMLP, the lipid mediators PAF and LTB4, and calcium ionophore A23187. Whereas we found that A23187 produced a large increase in eosinophil Mac-1 expression, the eosinophil responses to PAF and $\mathrm{C} 5 \mathrm{a}$ were relatively modest, considering the potent effect that both of these substances have on eosinophil chemotaxis (4144). A similar pattern of eosinophil response was reported by Giembycz et al. (45), who found that A23187 produced a many- 
fold larger release of arachidonic acid metabolites than did PAF or C5a. The mechanisms responsible for the relative unresponsiveness of Mac-1 up-regulation on eosinophils stimulated with C5a or PAF are not known, but could involve differences in the receptors for $\mathrm{C} 5 \mathrm{a}$ and $\mathrm{PAF}$ or in signal transduction mechanisms. The eosinophil receptor for $\mathrm{C} 5 \mathrm{a}$ has recently been shown to be distinct from the neutrophil C5a receptor (46). Because eosinophils lack a high-affinity receptor for $\operatorname{FMLP}(22,47)$, the minimal up-regulation of eosinophil Mac-1 in response to FMLP was expected, and this result confirmed the interpretation we made in a previous study, where distinct peaks corresponding to eosinophils and neutrophils were observed in the Mac-1 distributions of FMLP-stimulated granulocytes from fetuses with $\mathrm{Rh}$ disease (12).

We failed to find any effect of the ECFA tetrapeptides or histamine on eosinophil expression of either Mac-1 or CR1. These substances were reported to be chemotactic for eosinophils and to enhance the rosetting of eosinophils with $\mathrm{C} 3 \mathrm{~b}$-coated erythrocytes $(48,49)$. A later study did not substantiate a significant chemotactic effect, however, and their effect on eosinophil rosetting does not appear to have been reinvestigated (42). Our data do not support a role for a direct effect of these substances on up-regulation of eosinophil complement receptors.

In conclusion, we have shown that cord blood eosinophils from healthy neonates at term have a defect in quantitative upregulation of Mac-1 expression similar to that of neonatal neutrophils. It is not yet known if this in vitro finding has independent functional significance, or whether neonatal eosinophils, like neutrophils, also have qualitative abnormalities in Mac-1-dependent functions. Further studies are needed to determine if neonatal eosinophils have impairments in adherence, transendothelial migration, and chemotaxis, and, if so, whether the underlying mechanisms are the same as in neonatal neutrophils.

Acknowledgments. The authors thank Ingrid Schmid and Valentin Isacescu, M.D., for expert assistance with flow cytometry data collection and Daphne Walker, M.D., for assistance in obtaining blood specimens.

\section{REFERENCES}

1. Anderson DC, Springer TA 1987 Leukocyte adhesion deficiency: an inherited defect in the Mac-1, LFA-1, and p150,95 glycoproteins. Annu Rev Med 38:175-194

2. Arnaout MA 1990 Structure and function of the leukocyte adhesion molecules CD11/CD18. Blood 75:1037-1050

3. Carlos TM, Harlan JM 1990 Membrane proteins involved in phagocyte adherence to endothelium. Immunol Rev 114:5-28

4. Springer TA 1990 Adhesion receptors of the immune system. Nature 356:425434

5. Todd RF III, Arnaout MA, Rosin RE, Crowley CA, Peters WA, Babior BM 1984 Subcellular localization of the large subunit of Mol (Mol-alpha; formerly gp 110), a surface glycoprotein associated with neutrophil adhesion. $\mathrm{J}$ Clin Invest 74:1280-1290

6. Berger M, O'Shea J, Cross AS, Folks TM, Chused TM, Brown EJ, Frank MM 1984 Human neutrophils increase expression of $\mathrm{C} 3 \mathrm{bi}$ as well as $\mathrm{C} 3 \mathrm{~b}$ receptors upon activation. $J$ Clin Invest 74:1566-1571

7. Vedder NB, Harlan JM 1988 Increased surface expression of CD $11 \mathrm{~b} / \mathrm{CD} 18$ (Mac-1) is not required for stimulated neutrophil adherence to cultured endothelium. J Clin Invest 81:676-682

8. Wilson CB 1986 Immunological basis for increased susceptibility of the neonate to infection. J Pediatr 108:1-12

9. Hill HR 1987 Biochemical, structural, and functional abnormalities of polymorphonuclear leukocytes in the neonate. Pediatr Res 22:375-382

10. Bruce MC, Baley JE, Medvik KA, Berger M 1987 Impaired surface membrane expression of $\mathrm{C} 3 \mathrm{bi}$ but not $\mathrm{C} 3 \mathrm{~b}$ receptors on neonatal neutrophils. Pediatr Res 21:306-311

11. Anderson DC, Freeman KLB, Heerdt B, Hughes BJ, Jack RM, Smith CW 1987 Abnormal stimulated adherence of neonatal granulocytes: impaired induction of surface MAC-1 by chemotactic factors or secretagogues. Blood 70:740-750

12. Smith JB, Campbell DE, Ludomirsky A, Polin RA, Douglas SD, Garty BZ Harris MC 1990 Expression of the complement receptors CR 1 and CR 3 and the type III $\mathrm{Fc} \gamma$ receptor on neutrophils from newborn infants and from fetuses with $\mathrm{Rh}$ disease. Pediatr Res 28:120-126

13. Anderson DC, Rothlein R, Marlin SD, Krater SS, Smith CW 1990 Impaired transendothelial migration by neonatal neutrophils: abnormalities of Mac-1 (CD11b/CD18)-dependent adherence reactions. Blood 76:2613-2621
14. Anderson DC Abbassi O Kishimoto TK Mclntire LV, Smith CW 1990 Diminished human MEL-14 antigen on neonatal neutrophils underlies impaired CD11/CD18-independent adhesion to endothelial cells. Pediatr Res 27:153A(abstr)

15. Yazdanbakhsh M. Eckmann CM, Roos D 1985 Characterization of the interaction of human eosinophils and neutrophils with opsonized particles. $J$ Immunol 135:1378-1384

16. Fischer E, Capron M, Prin L, Kusinerz JP, Kazatchkine MD 1986 Human eosinophils express CR1 and CR3 complement receptors for cleavage fragments of C3. Cell lmmunol 97:297-306

17. Hartnell A, Moqbel R, Walsh GM, Bradley B, Kay AB $1990 \mathrm{Fc} \gamma$ and CD11/ $\mathrm{CD} 18$ receptor expression on normal density and low density human eosinophils. Immunology 69:264-270

18. Slungaard A, Vercellotti GM, Walker G, Nelson RD, Jacob HS 1990 Tumor necrosis factor $\alpha /$ cachectin stimulates eosinophil oxidant production and toxicity towards human endothelium. J Exp Med 171:2025-2041

19. Thorne KJI, Richardson BA, Mazza G, Butterworth AE 1990 A new method for measuring eosinophil activating factors, based on the increased expression of CR3 $\alpha$-chain (CD1 1b) on the surface of activated eosinophils. J Immunol Methods 133:47-54

20. Lamas AM, Mulroney CM, Schleimer RP 1988 Studies on the adhesive interaction between purified human eosinophils and cultured vascular endothelial cells. J Immunol 140:1500-1505

21. Walsh GM, Harnell A, Wardlaw AJ, Kurihara K, Sanderson CJ, Kay AB 1990 IL-5 enhances the in vitro adhesion of human eosinophils, but not neutrophils, in a leucocyte integrin (CD11/18)-dependent manner. Immunology 71:258-265

22. Roberts RL, Gallin JI 1985 Rapid method for isolation of normal human peripheral blood eosinophils on discontinuous Percoll gradients and comparison with neutrophils. Blood 65:433-440

23. Terstappen LWMM. Hollander Z, Meiners H. Loken MR 1990 Quantitative comparison of myeloid antigens on five lineages of mature peripheral blood cells. J Leukocyte Biol 48:138-148

24. Fearon DT, Collins LA 1983 Increased expression of C3b receptors on polymorphonuclear leukocytes induced by chemotactic factors and by purification procedures. J Immunol 130:370-375

25. Jones DH, Schmalstieg FC, Kempsey K, Krater SS. Nannen DD, Smith CW, Anderson DC 1990 Subcellular distribution and mobilization of MAC-1 (CD1 1b/CD18) in neonatal neutrophils. Blood 75:488-498

26. Gibson EL, Vaucher Y, Corrigan JJ 1979 Eosinophilia in premature infants: relationship to weight gain. J Pediatr 95:99-101

27. Bhat AM, Scanlon JW 1981 The pattern of eosinophilia in premature infants. J Pediatr 98:612-6:16

28. Kulczycki Jr A 1984 Human neutrophils and eosinophils have structurally distinct Fc gamma receptors. J Immunol 133:849-854

29. Looney RJ, Ryan DH, Takahashi K, Fleit HB, Cohen HJ, Abraham GN, Anderson $\mathrm{CL} 1986$ Identification of a second class of $\mathrm{IgG}$ receptors on human neutrophils: a 40 kilodalton molecule also found on eosinophils. J Exp Med 163:826-836

30. Graziano RF, Looney SJ, Shen L, Fanger MW 1989 Fc $\gamma$ R-mediated killing by eosinophils. J Immunol 142:230-235

31. Hansel TT, Pound JD, Pilling D, Kitas GD, Salmon M, Gentle TA, Lee SS, Thompson RA 1989 Purification of human blood eosinophils by negative selection using immunomagnetic beads. J Immunol Methods 122:97-103

32. Kunjummen RD, Raghavender BH, Walker DK, Smith JB 1990 Neonata eosinophils have a defect in $\mathrm{CD} 1 \mathrm{lb} / \mathrm{CD} 18$ expression similar to neonatal neutrophils. Pediatr Res 27:267A(abstr)

33. Hansel TT, Pound JD. Thompson RA 1990 Isolation of eosinophils from human blood. $J$ Immunol Methods 127:153-164

34. Ory PA, Goldstein IM, Kwoh EE, Clarkson SB 1989 Characterization of polymorphic forms of $\mathrm{Fc}$ receptor III on human neutrophils. J Clin Invest 83:1676-1681

35. Huizinga TWJ, van der Shoot CE, Jost C, Klaassen R, Kleijer M, von dem Borne AEGKr, Roos D, Tetteroo PAT 1988 The PI-linked receptor FcRIII is released on stimulation of neutrophils. Nature 333:667-669

36. Selvaraj P, Rosse WF, Silber R, Springer T 1988 The major Fc receptor in blood has a phosphatidylinositol anchor and is deficient in paroxysmal nocturnal hemoglobinuria. Nature 333:565-567

37. Boros P, Gardos E, Bekesi GJ, Unkeless JC 1990 Change in expression of Fc $\gamma$ RIII (CD16) on neutrophils from human immunodeficiency virus-infected individuals. Clin Immunol Immunopathol 54:281-289

38. Strauss RG, Snyder EL 1983 Activation and activity of the superoxidegenerating system of neutrophils from human infants. Pediatr Res 17:662664

39. Ambruso DR, Stork LC, Gibson BE, Thurman GW 1987 Increased activity of the respiratory burst in cord blood neutrophils: kinetics of the NADPH oxidase enzyme system in subcellular fractions. Pediatr Res 21:205-210

40. Fletcher MP, Seligmann BE 1985 Monitoring human neutrophil granule secretion by flow cytometry: secretion and menbrane potential assessed by light scatter and a fluorescent probe of membrane potential. J Leukocyte Biol 37:431-447

41. Klebanoff SJ, Durack DT, Rosen H, Clark RA 1977 Functional studies on human peritoneal eosinophils. Infect Immun 17:167-173

42. Wardlaw AJ, Moqbel R. Cromwell O, Kay AB 1986 Platelet activating factor: a potent chemotactic and chemokinetic factor for human eosinophils. J Clin Invest 78:1701-1706 
43. Hayashi K 1989 Comparative study on the responses of human neutrophils and eosinophils to platelet-activating factor. J Clin Biochem Nutr 6:77-86

44. Yukawa T, Kroegel C, Evans P, Fukuda T, Chung KF, Barnes PJ 1989 Density heterogeneity of eosinophil leukocytes: induction of hypodense eosinophils by platelet-activating factor. Immunology $68: 140-143$

45. Giembycz MA, Kroegel C, Barnes PJ 1990 Platelet activating factor stimulates cyclooxygenase activity in guinea pig eosinophils. J Immunol 144:34893497

46. Gerard NP, Hodges MK, Drazen JM, Weller PF, Gerard C 1989 Characteriza- tion of a receptor for C5a anaphylatoxin on human eosinophils. J Biol Chem 264:1760-1766

47. Koenderman L, Kok PTM, Hamelink ML, Verhoeven AJ, Bruijnzeel PLB 1988 An improved method for the isolation of eosinophilic granulocytes from peripheral blood of normal individuals. J Leukocyte Biol 44:79-86

48. Anwar ARE, Kay AB 1977 The ECF-A tetrapeptides and histamine selectively enhance human eosinophil complement receptors. Nature 269:522-524

49. Anwar ARE, Kay AB 1978 Enhancement of human eosinophil complement receptors by pharmacological mediators. Infect Immun 121:1245-1250

\section{Announcements}

\section{Call for Abstracts}

The American Pediatric Society and The Society for Pediatric Research announce the abstract deadline for the 1992 Annual Meeting (May 4-7, 1992, Convention Center, Baltimore, MD) has been set as January 3, 1992. For further information, contact: APS/SPR, 141 Northwest Point Blvd., P.O. Box 675, Elk Grove Village, IL 60009-0675, (708)427-0205, FAX (708)427-1305. 